\title{
Anginal pain and elevated troponin level despite normal coronary angiography: hypertrophic cardiomyopathy with severe obstruction due to vasodilator/diuretic therapy for coincident arterial hypertension
}

\author{
Adam Gębka ${ }^{1}$, Renata Rajtar-Salwa ${ }^{1}$, Rafał Hładij ${ }^{1}$, Paweł Petkow Dimitrow ${ }^{1,2}$ \\ ${ }^{1}$ Second Department of Cardiology and Cardiovascular Interventions, University Hospital, Krakow, Poland \\ ${ }^{2}$ Second Department of Cardiology, Jagiellonian University Medical College, Krakow, Poland
}

Adv Interv Cardiol 2018; 14, 1 (51): 109-111 DOI: https://doi.org/10.5114/aic.2018.74367

A 69-year-old female patient with dyspnea (NYHA III), chest pain (CCS II), and an elevated high-sensitivity troponin I (hs-Tnl) level (274 ng/l in the face of normal creatinine - $78 \mu \mathrm{mol} / \mathrm{l})$ was admitted to our department. She had a history of arterial hypertension (max. 190/ $140 \mathrm{~mm} \mathrm{Hg}$ ) and hypercholesterolemia. ECG revealed sinus rhythm of 75 beats per minute, ST-segment elevation in $\mathrm{V} 2$ up to $2 \mathrm{~mm}$ and a negative T wave in V2-V6. On current (three drugs) pharmacotherapy consisting of an angiotensin receptor blocker, a diuretic and a very low dose of metoprolol (25 mg/day), blood pressure was $112 / 61 \mathrm{~mm} \mathrm{Hg}$ (the relatively low value for arterial hypertension was probably related to left ventricular outflow tract (LVOT) obstruction; see echocardiogram description below).

Echocardiography was performed prior to coronary angiography. Current angina pectoris, with elevated troponin level, ischemic signs in ECG and risk factors of coronary artery disease - age 69 years, smoking and hypertension - were arguments for the decision to perform coronary angiography (on the admission day, both echocardiography and coronary angiography were performed). Echocardiography revealed significantly reduced left ventricle (LV) cavity size with akinesia of the apex and the presence of thrombus (Figure $1 \mathrm{~A}$ ). Asymmetric LV hypertrophy (LV end-diastolic septal thickness of $2.0 \mathrm{~cm}$ ) and severe LVOT gradient of $85 \mathrm{~mm} \mathrm{Hg}$ (Figure $1 \mathrm{~B}$ ) with systolic anterior movement (SAM) of mitral leaflet in resting condition were detected as a common picture of hypertrophic cardiomyopathy (HCM) (Figure $1 \mathrm{C}$ ). Thick mitral valve leaflets are a frequent phenomenon in $\mathrm{HCM}$ as well as a early phenomenon of genotype posi- tive but phenotype negative HCM patients. The coronary artery angiogram was normal in left side epicardial coronary vasculature, and nearly normal with insignificant stenosis of the right coronary artery.

The vasodilator and diuretic were gradually decreased (to maximize LV preload) and were substituted by increasing doses of $\beta$-blocker. Simultaneously both LVOT gradient and hs-Tnl level decreased in day-by-day monitoring to normal values (Figure $1 \mathrm{D}$, final levels: $\mathrm{hs}-\mathrm{Tnl}=$ $8.4 \mathrm{ng} / \mathrm{l}$ and LVOT gradient $=12 \mathrm{~mm} \mathrm{Hg}$ ). The time profile of the NT-proBNP level declined but the last measurement was still markedly above the normal value (admission - $5789 \mathrm{pg} / \mathrm{ml}, 3^{\text {rd }}$ day - $2415 \mathrm{pg} / \mathrm{ml}$, discharge $541 \mathrm{pg} / \mathrm{ml}$ ). Anginal pain and dyspnea gradually decreased. Finally, at discharge, the patient was in NYHA class II, without angina pectoris, ECG changes were stable during hospitalization and at discharge, the latest blood pressure was $124 / 65 \mathrm{~mm} \mathrm{Hg}$ (at a dose of only one drug - $100 \mathrm{mg}$ of metoprolol). 24-hour ECG Holter monitoring, performed on the $2^{\text {nd }} / 3^{\text {rd }}$ day when positive hs-Tnl was detected, revealed one very short episode of non-sustained ventricular tachycardia (nsVT). Neither palpitation nor syncope in the pre-hospitalization period was present, but full exclusion of nsVT without ECG (especially Holter monitoring just before admission) recording is unrealistic. The family history for sudden cardiac death was negative.

According to the third universal definition of myocardial infarction, lack of ECG changes or a normal coronary angiogram does not exclude acute coronary syndrome (ACS). Our patient presented hypertrophic cardiomyopathy with severe LVOT obstruction as a variant of ACS (type 2). Myocardial ischemia was induced by imbalance

\section{Corresponding author:}

Paweł Petkow Dimitrow MD, PhD, Second Department of Cardiology, Jagiellonian University Medical College, 17 Kopernika St, $31-501$ Krakow, Poland, phone: +48 12 4247170, e-mail: dimitrow@mp.pl

Received: 27.10.2017, accepted: 29.12.2017. 

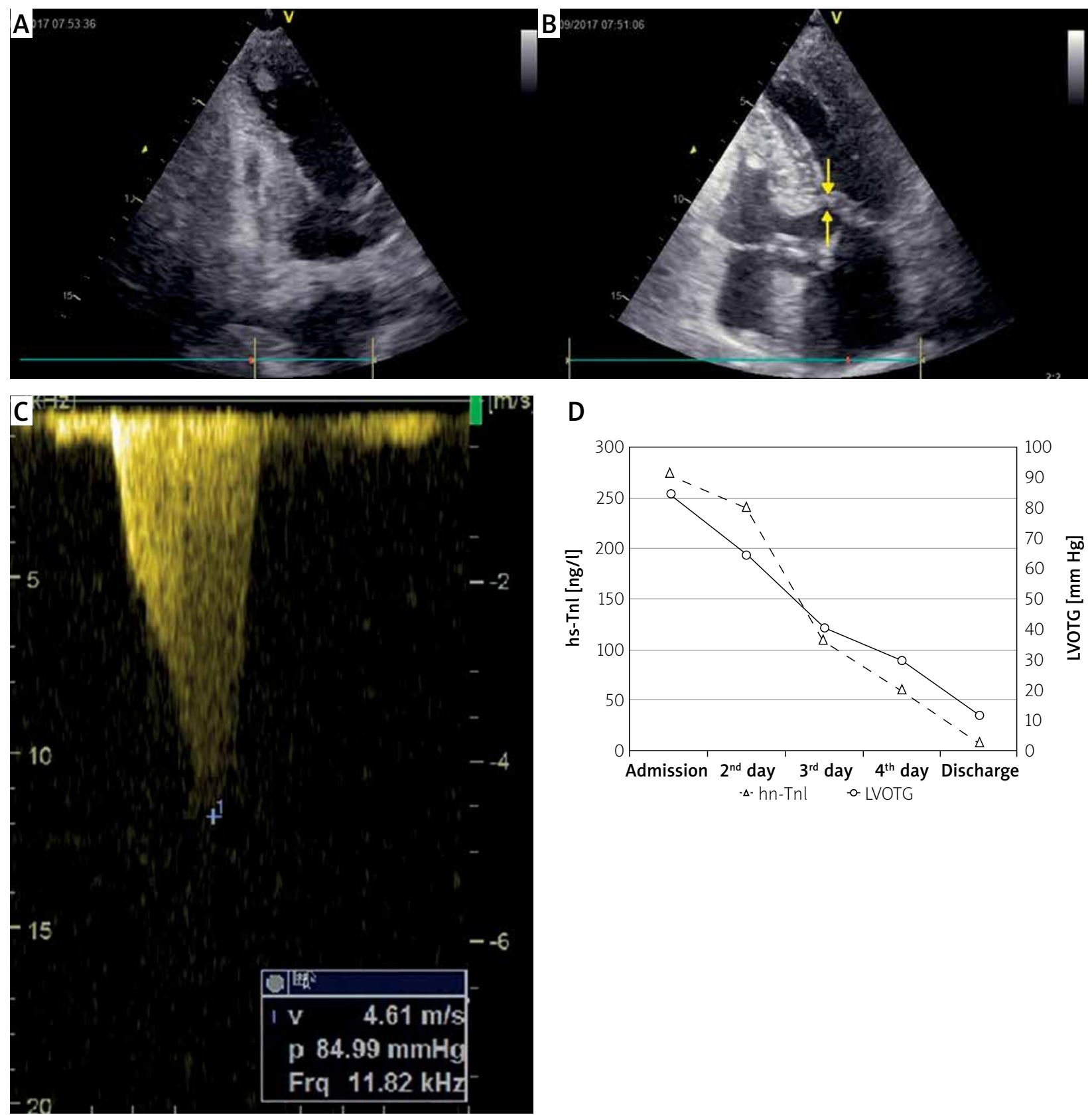

\section{D}

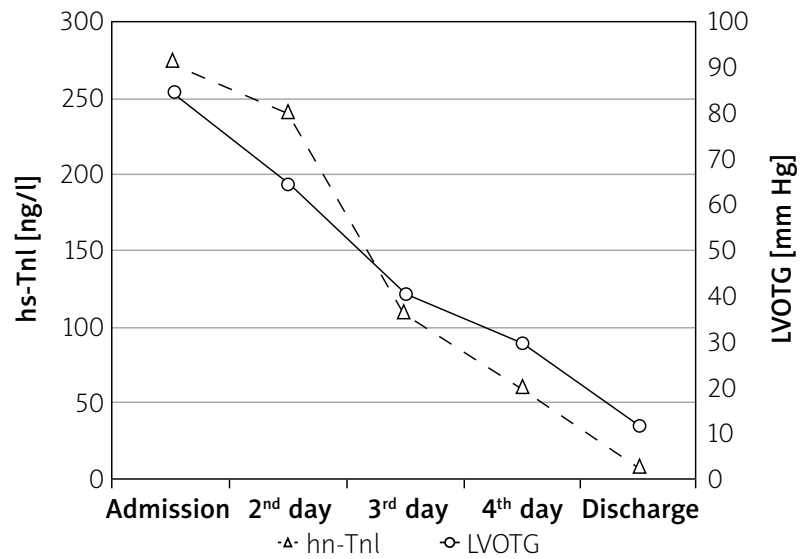

Figure 1. A - Thrombus in left ventricle, B - left ventricular outflow tract obstruction confirmed by an echocardiographic examination (full septal - leaflet contact at systole - red arrows), C - LVOT gradient $85 \mathrm{~mm} \mathrm{Hg}$, D - time profile of hs-Tnl and left ventricular outflow tract gradient decreasing in parallel

between oxygen supply/demand (increased demand due to high LV systolic pressure, generated by significant LVOT gradient). The patient did not present symptoms and signs suggesting acute heart failure (it is important that a beneficial decrease of NT-proBNP level occurred despite termination of the diuretic and angiotensin receptor inhibitor) or pulmonary embolism (mild tricuspid regurgitation, without pulmonary hypertension, normal sized right ventricle in echocardiography).

As regards ventricular arrhythmia, briefly, a single episode of nonsustained VT was recorded during hospi- talization. During the remaining time of hospitalization continuous ECG monitoring did not reveal nsVT. Before admission, the patient denied palpitation of heart or syncope (she was probably without an episode of nsVT/sVT).

In our opinion, elimination of drugs reducing preload (valsartan $160 \mathrm{mg}$ and hydrochlorothiazide $12.5 \mathrm{mg}$ ), lowered afterload due to valsartan action and increasing doses of inotropic acting $\beta$-blocker $(100 \mathrm{mg}$ ) are responsible for LVOT gradient reduction and coincidence with troponin normalization. In contrast, NT-proBNP did not reach a normal value. Stress echocardiography to pro- 
voke a maximal LVOT gradient was not performed due to the presence of apical thrombus in the LV.

The presented case shows a relationship between decreasing hs-Tnl level and reducing LVOT gradient. This suggests that an increased LVOT gradient may be partially responsible for myocardial ischemia (apart from tachycardia diagnosed to be responsible for hs-Tnl release in hypertrophic cardiomyopathy) [1, 2]. In a previous report, HCM patients with elevated hs-Tnl levels (positive hsTnl test) had higher values of both resting and provoked LVOT gradient [3]. However, this analysis was based only on one measurement of LVOT gradient and hs-Tnl synchronized in a 24-hour period. The observation provides five pairs of simultaneous measurements of LVOT gradient and hs-Tnl at different time points, revealing the time-course profile of LVOT gradient and ischemic biomarker parameters.

Previously, in invasive studies (resting supine position) myocardial ischemia (lactate production) corresponding with an increasing LVOT gradient was induced by rapid atrial pacing or $\beta$-receptor stimulation $[4,5]$. Beta-receptor stimulation increased the LVOT gradient in parallel with release of an ischemic biomarker, i.e., lactate production [4]. In contrast, surgical decrease of the LVOT gradient was correlated with a beneficial shift from lactate production to consumption [6].

In conclusion, our case has shown that normalization of the LVOT gradient was in parallel with normalization of hs-Tnl, while a high value of NT-proBNP was not reduced to the normal range.

This observation, showing that hs-Tnl is more related to LVOT gradient than NT-proBNP, supports the hypothesis that ischemic markers may better reflect the risk of sudden cardiac death in HCM than natriuretic biomarkers [7].

\section{Conflict of interest}

The authors declare no conflict of interest.

\section{References}

1. Hładij R, Rajtar-Salwa R, Dimitrow PP. Association of elevated troponin levels with increased heart rate and higher frequency of nonsustained ventricular tachycardia in hypertrophic cardiomyopathy. Pol Arch Intern Med 2017; 126: 445-7.

2. Rajtar-Salwa R, Dimitrow PP, Miszalski-Jamka T. Role of cardiac magnetic resonance in differentiating between acute coronary syndrome and apical hypertrophic cardiomyopathy. Adv Interv Cardiol 2016; 12: 380-2.

3. Hładij R, Rajtar-Salwa R, Dimitrow PP. Troponin as ischemic biomarker is related with all three echocardiographic risk factors for sudden death in hypertrophic cardiomyopathy (ESC Guidelines 2014). Cardiovasc Ultrasound 2017; 15: 24.

4. Udelson JE, Cannon RO $3^{\text {rd }}$, Bacharach SL, et al. Beta-adrenergic stimulation with isoproterenol enhances left ventricular diastolic performance in hypertrophic cardiomyopathy despite potenti- ation of myocardial ischemia. Comparison to rapid atrial pacing. Circulation 1989; 79: 371-82.

5. Cannon RO $3^{\text {rd }}$, Schenke WH, Maron BJ, et al. Differences in coronary flow and myocardial metabolism at rest and during pacing between patients with obstructive and patients with nonobstructive hypertrophic cardiomyopathy. J Am Coll Cardiol 1987; 10: 53-62.

6. Cannon RO $3^{\text {rd }}$, McIntosh CL, Schenke WH, et al. Effect of surgical reduction of left ventricular outflow obstruction on hemodynamics, coronary flow, and myocardial metabolism in hypertrophic cardiomyopathy. Circulation 1989; 79: 766-75.

7. Rajtar-Salwa R, Hładij R, Dimitrow PP. Elevated level of troponin but not $\mathrm{N}$-terminal-pro-brain natriuretic peptide is associated with increased risk of sudden cardiac death in hypertrophic cardiomyopathy calculated according to ESC Guidelines 2014. Dis Markers 2017; 2017: 9417908. 